Anaesthesist 2010 · 59:1075

DOI 10.1007/s00101-010-1833-4

(c) Springer-Verlag 2010

\author{
V. Dörges \\ Klinik für Anästhesiologie und Operative Intensivmedizin, \\ Universitätsklinikum Schleswig-Holstein, Campus Kiel
}

\title{
Wissenschaft besiegt Politik und Kommerz
}

Die neuen Leitlinien zur kardiopulmonalen Reanimation („,cardiopulmonary resuscitation", CPR) sind in einem sehr umfangreichen Prozess mit weltweiter Beteiligung von Experten entstanden [3, 4]. So waren die Reanimationsfachgesellschaften aus allen globalen Regionen vertreten, was wiederum eine hervorragende Legitimität des Endprodukts sichert. Dies ist gar nicht hoch genug zu bewerten, weil unterschiedliche (gewachsene) Behandlungsstrategien, kulturelle Sichtweisen und Arbeitsbedingungen in den verschiedensten Winkeln der Erde ein gemeinsames Vorgehen oft erschweren oder sogar unmöglich machen. Bei den vorliegenden Leitlinien wurde sehr streng darauf geachtet, einen Interessenkonflikt der beteiligten Experten zu verhindern, um ein „Schönreden“ der Daten nicht zu ermöglichen.

Zum Beispiel durfte kein Delegierter von ihm untersuchte Techniken, Medikamente oder Geräte bewerten - so haben Defibrillationsexperten die Notfallbeatmung bewertet und umgekehrt. Bei dieser Strategie muss zwar Detailwissen u. U. erst erarbeitet werden, aber letztendlich zählt die große Linie - was belegen große, prospektiv randomisierte Studien? Nur so kann eine nachhaltige Verbesserung der CPR-Leitlinien erzielt werden.

Der kritische Leser sollte immer nach dem Prozess hinter jeglichen Empfehlungen fragen, um zu verhindern, dass ärztliche Behandlungsstrategien (und damit häufig Klinikbudgets) von diversen Interessengruppen des „medizinisch-industriellen Komplexes" gesteuert werden. So wurde z. B. die erste Ausgabe der „Surviving Sepsis Campaign“ [1] sehr scharf von Wissenschaftlern des Amerikanischen

National Institute of Health verurteilt, weil Daten von Studien mit aktiviertem Protein C nicht korrekt beurteilt worden waren [2]. Auch die Zeitschrift Critical Care Medicine hatte ihre Sorgfaltspflicht bei der kritischen Prüfung der zur Veröffentlichung vorgeschlagenen Manuskripte dabei zumindest teilweise aus den Augen verloren, was aber ein Kliniker ohne wissenschaftliche Ausbildung gar nicht beurteilen kann.

Umso mehr kann ich hier feststellen, dass die CPR-Leitlinien eine Eliteliga der Erstellung von Leitlinien geschaffen haben - weltweite Experten ohne Interessenkonflikt haben evidenzbasierte Leitlinien geschaffen - die „Währung“ dabei sind signifikante Ergebnisse aus klinischen Studien. Wissenschaft besiegt Politik und Kommerz; es wäre schön, wenn es immer so wäre.

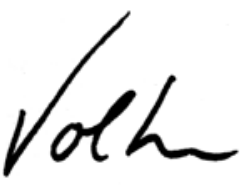

V. Doerges

\section{Korrespondenzadresse}

Prof. Dr. V. Dörges

Klinik für Anästhesiologie und Operative Intensivmedizin, Universitätsklinikum Schleswig-Holstein, Campus Kiel Arnold-Heller-Straße 3, Haus 12, 24105 Kiel doerges@uk-sh.de

Interessenkonflikt. Der Autor hat keine Interessenkonflikte, die im Zusammenhang mit dem Inhalt dieses Editorials oder des Beitrags zu den CPR-Leitlinien stehen.

\section{Literatur}

1. Dellinger RP, Carlet JM, Masur H et al (2004) Surviving Sepsis Campaign guidelines for management of severe sepsis and septic shock. Crit Care Med 32:858-873

2. Eichacker $P Q$, Natanson C, Danner RL (2006) Surviving sepsis - practice guidelines, marketing campaigns, and Eli Lilly. N Engl J Med 355:1640-1642

3. Nolan JP, Hazinski MF, Billi JE et al (2010) Part 1: Executive summary: 2010 international consensus on cardiopulmonary resuscitation and emergency cardiovascular care science with treatment recommendations. Resuscitation 81 (Suppl 1):e1-25

4. Wenzel V, Russo S.G, Arntz HR et al (2010) Kommentar zu den Leitlinien 2010 zur kardiopulmonalen Reanimation des European Resuscitation Council. Anaesthesist 59:1105-1123 